Advances in High-Pressure Technology for Geophysical Applications

Jiahua Chen, Yanbin Wang, T.S. Duffy, Guoyin Shen, L.F. Dobrzhinetskaya, editors

(C) 2005 Elsevier B.V. All rights reserved.

\title{
Chapter 21 \\ Calibration based on a primary pressure scale in a multi-anvil device
}

\author{
Hans J. Mueller, Frank R. Schilling, Christian Lathe \\ and Joern Lauterjung
}

\begin{abstract}
A key question to all high-pressure research arises from the reliability of pressure standards. There is some indication and discussion of an uncertainty of 10-20\% for higher pressures in all standards. Independent and simultaneous investigation of the dynamical (ultrasonic interferometry of elastic wave velocities) and static (XRD-measurement of the pressure-induced volume decline) compressibility on a sample reveal the possibility of a standard-free pressure calibration and, consequently an absolute pressure measurement, because all required parameter are collected directly; no additional data, e.g. the volume dependence of the Grüneisen parameter etc. are needed. Ultrasonic interferometry is used to measure velocities of elastic compressional and shear waves in the multi-anvil high-pressure device MAX80 at HASYLAB Hamburg enables XRD, $X$-radiography, and ultrasonic experiments. Two of the six anvils were equipped with lithium niobate transducers of $33.3 \mathrm{MHz}$ natural frequency. $\mathrm{NaCl}$ was used as pressure calibrant, using the equation of state (EoS) of [J. Appl. Phys. 42 (1971) 3239], and sample for ultrasonic interferometry at the same time. From the ultrasonic wave velocity data, $v_{p}$ and $v_{s}$, we calculated the compressibility of $\mathrm{NaCl}$ as a function of pressure independent from $\mathrm{NaCl}$-pressure calibrant. To derive the ultrasonic wave velocities from the interferometric frequencies of constructive and destructive interference requires precise in situ sample length measurements. For a NaCl-sample this is of particular importance, because the sample is the most ductile part of the whole set-up. We measured the sample length by XRD-scanning and by X-radiography. The compressibility results, derived from the ultrasonic data, were compared with data of static compression experiments up to 5 GPa [Phys. Rev. 57 (1940) 237] and up to 30 GPa [J. Geophys. Res. 91 (1986) 4949] using experimental data from [J. Phys. Chem. Solids 41 (1980) 517] and [Accurate Characterization of the High Pressure Environment]. At 1.2 and $5.3 \mathrm{GPa}$ our velocity-derived compressibility data agree with the results of static compression. In the range between 2 and $4 \mathrm{GPa}$ our dynamical data have 1.5-3\% higher values. In general, the pressure revealed according to [J. Appl. Phys. 42 (1971) 3239] is in accordance to our standard-free pressure calibration. Consequently, up to $8 \mathrm{GPa}$ the $\mathrm{NaCl}$ pressure standard has a reliability of at least 1\%. However, there is some evidence that at higher pressures the inaccuracy of the $\mathrm{NaCl}$ standard seems to exceed 1\%. Extrapolation of the compressibility data to higher pressures would also result in an increasing deviation, for EoS-fit and numerical fit of the density more than for the deformation fit.
\end{abstract}

\section{Introduction}

Multi-anvil devices are a very successful tool for experimental simulation of mantle conditions with relatively large samples. Accurate pressure determinations are critical to 
most high-pressure measurements. However, pressure calibration and the reliability of pressure standards are discussed controversially.

The formation and the development of gaskets between the anvils causes a deviation between load per anvil surface and pressure inside the set-up because of friction, material variation of the pressure transmitting medium, minor fit variation in the set-up, minor adjustment variation of the set-up and the anvils to each other, and different compressibility of the samples. Recent pressure determinations in a gas piston-cylinder apparatus successfully reduced the uncertainty to $0.2 \%$, which is as low as that of free piston gages at $2.5 \mathrm{GPa}$ (Getting, 1998). Therefore, in situ pressure measurements and precise standards are very important for this type of experiment.

Different options for pressure calibration exist

- using the known pressure of mineral reactions due to phase transitions, e.g. by measuring the change of electrical conductivity or using petrological experiments to determine mineral reactions. Several discrete measurements result in a pressure calibration curve (Luth, 1993),

- spectroscopic observation of a pressure-dependent absorption band or peak, e.g. ruby chip (Piermarini et al., 1975; Mao et al., 1986) (standard method for diamond anvil cells, not suitable for multi-anvil cells),

- continuous determination of the pressure-dependent unit cell size of a standard by X-ray diffraction, using the pressure marker's equation of state (EoS) (Decker, 1971; Chen et al., 2000).

The most common material to calibrate for conditions simulating the upper mantle is $\mathrm{NaCl}$, following the EoS published by Decker (1971), recently revised by Brown (1999). At the time that Decker made his calculations, the EoS was based on first principles and therefore as independent as possible. Ruby fluorescence is a secondary pressure scale and is usually calibrated against $\mathrm{NaCl}$ at less high pressures. Progress in indirect pressure scale measurements has led to precision, which exceeds the accepted uncertainty of the practical pressure scale by a factor of as much as five. A new indirect pressure scale would become available from the over-determination of the EoS of a reference material by simultaneous X-ray and ultrasonic measurements (Ruoff et al., 1973; Yoneda et al., 1994; Getting, 1998; Zha et al., 1998, 2000; Bassett et al., 2000).

MAX80 is a single-stage multi-anvil apparatus (Yagi, 1988) equipped for ultrasonic interferometry (Mueller et al., 2002, 2003) and permanently located at HASYLAB, Hamburg for having access to synchrotron radiation for in situ XRD measurements. We present simultaneous XRD- and high-pressure ultrasonic interferometry measurements of compressional and shear wave velocities of polycrystalline $\mathrm{NaCl}$ to determine a standard-free pressure scale and to test the existing EoS by Decker (1971) and Brown (1999). In situ sample length measurement, necessary for high-precision ultrasonic interferometry, were performed by scanning both sample interfaces to the adjacent buffer and reflector and evaluating the XRD-spectra, as well as by $\mathrm{X}$-radiography, i.e. taking X-ray shadow graphs of the set-up, recently installed at MAX80. 


\section{Techniques, methods, and materials description}

\subsection{Multi-anvil high-pressure apparatus MAX80}

MAX80 (Fig. 1) is a single-stage multi-anvil high-pressure apparatus with six tungsten carbide anvils to compress a cubic sample volume of maximum $8 \times 8 \times 8 \mathrm{~mm}^{3}$. The anvils are driven by a $2500 \mathrm{~N}$ uniaxial hydraulic ram, the top and bottom anvil directly, the lateral anvils by two load frames and four reaction bolsters, see Figure 2. Three anvil-sets with different truncations exist $-6,5,3.5 \mathrm{~mm}$. The maximum attainable pressures using $3.5 \mathrm{~mm}$ tungsten carbide anvils reach $12 \mathrm{GPa}$ at $2000 \mathrm{~K}$ produced by an internal graphite heater. The $6 \mathrm{~mm}$ truncation limits the maximum pressure to approximately $7 \mathrm{GPa}$.

Diffraction patterns are recorded in an energy-dispersive mode (XRD) using white X-rays from the storage ring DORIS III at HASYLAB. MAX80 is equipped with a germanium solid-state detector analyzing the diffracted white beam at a fixed angle with a resolution of $135 \mathrm{eV}$ for $6.3 \mathrm{keV}$ and $450 \mathrm{eV}$ for $122 \mathrm{keV}$. Using a double-crystal, fixedoffset monochromator with silicon (311) single crystals, calibrated in the wavelength range of $0.4-0.6 \AA$, and a $2048 \times 2048$ pixels CCD-camera angle-dispersive X-ray diffraction (not used in this study) is also available.

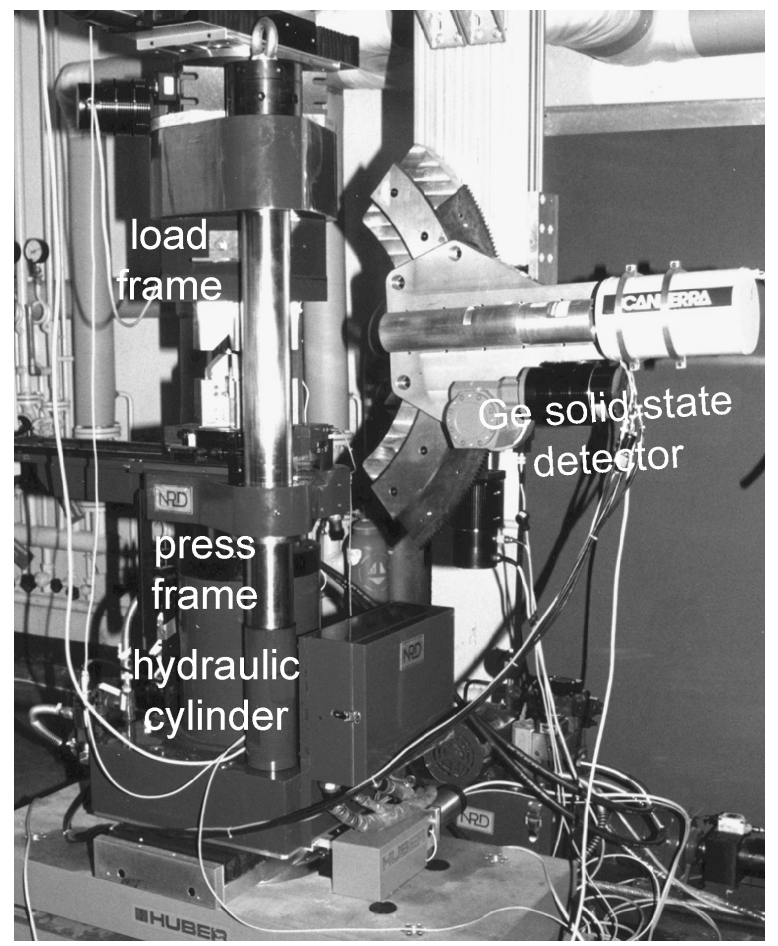

Figure 1. DIA-type multi-anvil apparatus MAX80 with Ge solid-state detector. The load frames are assembled at a 250 tons hydraulic ram. The Ge solid-state detector is also assembled at the press frame and follows the adjustment of the whole apparatus in relation to the X-ray beam. 


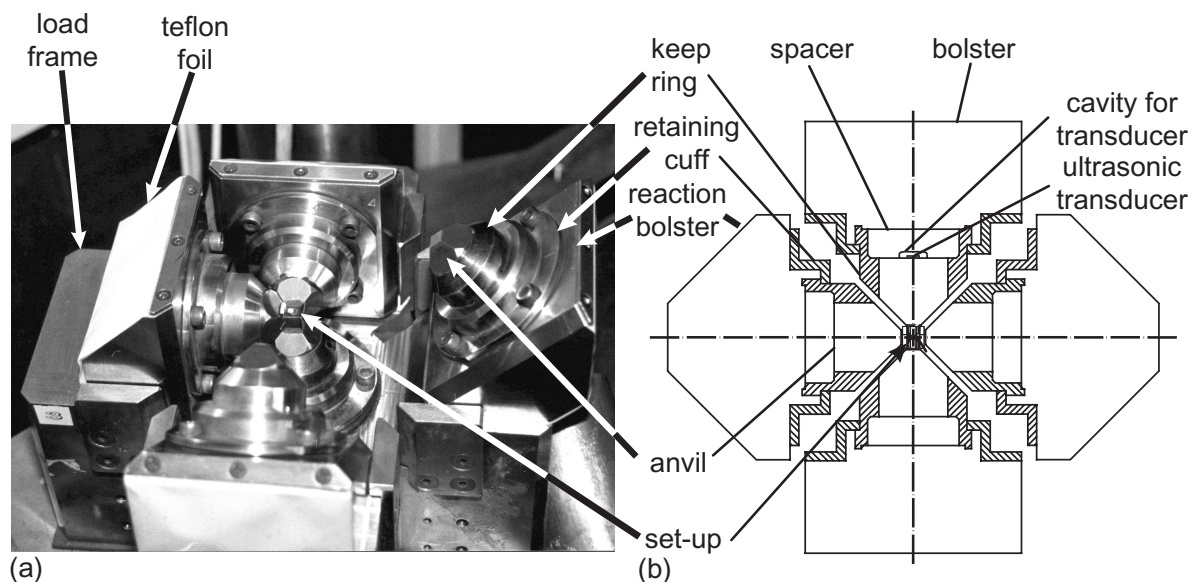

Figure 2. Load frame, anvils and sample arrangement of MAX80. (a) Apparatus opened for sample exchange and (b) vertical cross-section with transducer installation at the top anvil. The tungsten carbide anvils with a steel made keep ring are assembled to bolsters and reaction bolsters, respectively. Driven by the vertical movement of the hydraulic cylinder the load frame and the reaction bolsters generate the movement of the lateral anvils.

The pressure is measured by energy-dispersive XRD using the high-pressure EoS for $\mathrm{NaCl}$ (Decker, 1971). The method uses the observation of the elementary lattice cell compression of cubic $\mathrm{NaCl}$ crystals to derive the pressure in situ. These data are implemented in an in-house PC-program to calculate the resulting pressure at normal or given temperature. For details see Shimomura et al. (1985), Vaughan (1993), and Zinn et al. (1997).

In general, differential stress in the sample has the potential to affect the Decker scale. The first effect is that the volume change of $\mathrm{NaCl}$ might be overestimated. If the differential stress is greatest along the axis of their sample, then the added stress along this axis will also elastically shorten the sample resulting in a volume error that becomes interpreted as higher pressure. To estimate the value of differential stress, we performed a simple stress test by calculating the volume of the unit cell from 111 to 200 under high-pressure conditions. Generally, if the 111 suggests a smaller unit cell volume than the 200 , this would indicate a tendency to underestimate the sample volume. For run 3.27 we found a quotient of the unit cell volumes $V_{111} / V_{200}$ between +0.03 and $+0.25 \%$, i.e. any significant differential stress resulting in negative quotients was not found. The only indication for minor differential stress we noticed is the decrease of the 111 intensity compared to normal conditions. Because of the very low strength of $\mathrm{NaCl}$ differential stress seems to be much less important than for mineral samples. Therefore, $\mathrm{NaCl}$ is widely used as pressure transmitting medium, e.g. in piston-cylinder apparatus.

The high-pressure cell consists of a cube made of epoxy resin mixed with amorphous boron with the weight ratio 1:4 for better compressive strength containing the ultrasonic configuration, the heater, the pressure standard, and the thermocouple. Although the graphite heater was not necessary for the experiments presented here it was not removed from the set-up for $6 \mathrm{~mm}$ anvil truncation to use the standard ultrasonic configuration of 


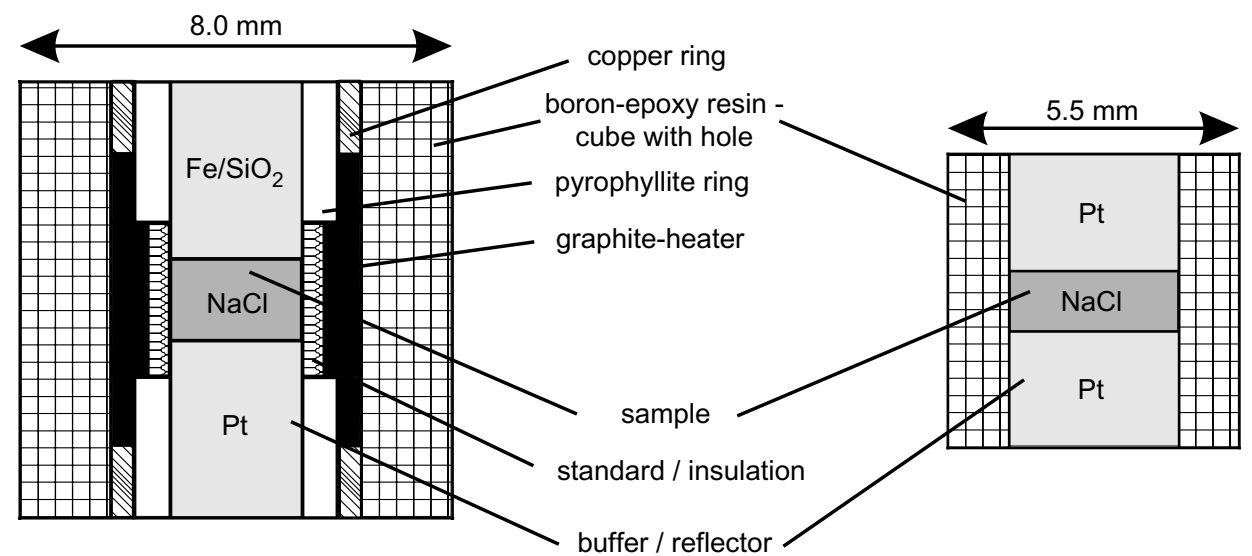

Figure 3. Ultrasonic high-pressure set-ups for anvil truncations of 6 and $3.5 \mathrm{~mm}$. The smaller set-up was not equipped with a heater to keep the sample cross-section as big as possible, because the strength of reflected ultrasonic waves is a function of the sample diameter.

MAX80 (Mueller et al., 2002). Removing the heater would result in pressure data not representative for the standard configuration. On the other hand, the $5 \mathrm{~mm}$ cube set-up was especially designed without heater to keep the sample surface bigger for stronger ultrasonic reflections, and to enhance the signal-to-noise-ratio. All interfaces between the sample and the close-fitting buffer rods/reflector bars are polished for optimal ultrasonic coupling (Fig. 3). Additional coupling media were not used. Copper rings contact the heater at the top and bottom anvils. The sample is surrounded by rings made from boron nitride or glass ceramics for electrical insulation and as a quasi-hydrostatic pressure transmitting medium. Further details of the apparatus are described by Mueller et al. Q1 (2004) on page xxx, this volume and also by Mueller et al. (2002).

\subsection{Ultrasonic interferometry}

Ultrasonic interferometry, using the interference between the incident and reflected waves inside the sample, was first described by McSkimin (1950). Piezoelectric transducers for the generation and detection of ultrasonic waves are cemented at the polished rear anvil's side outside the true pressure cell. One or two of the original MAX80 anvil spacers (see Figure 2) were replaced by redesigned parts for ultrasonic experiments. The new spacers have a cavity in their center to keep the ultrasonic transducer free of any stress. In principle, two types of ultrasonic set-ups were used in the presented experiments.

Asymmetrical set-ups are characterized by the optimization of buffers and reflectors, i.e. the buffer is made of a material resulting in intermediate acoustic impedance contrasts at both interfaces (anvil-buffer, buffer-sample) and the reflector material is selected for maximum reflection at the rear side of the sample. At ambient pressure, the reflection coefficient for the $\mathrm{NaCl}-\mathrm{Pt}$ interface is $80 \%$, the reflection coefficient for the $\mathrm{Pt}-\mathrm{TC}$ interface only $20 \%$. This means, that only a minor amount is reflected between anvil and 
platinum, but most energy is reflected between $\mathrm{NaCl}$ and $\mathrm{Pt}$, resulting in an optimized amplitude in the interference pattern. For the massive $\mathrm{NaCl}$ samples used in this study, powders were pressed, cut, and re-machined. Buffers made from iron, aluminum, and $\mathrm{Al}_{2} \mathrm{O}_{3}$ ceramics were used. Platinum was found to be the optimum reflector material. To measure at once, the velocity of compressional and shear waves simultaneously with asymmetrical set-ups, requires the assemblage of both $P$ - and $S$-wave transducers at one anvil or the use of a two-mode transducer as published by Kung et al. (2000). To ensure the maximum ultrasonic energy emission of the optimum cut transducers we used separate transducers for generation and detection, arranged in a circle as close as possible to one another (Fig. 4). The geometrical error introduced by the eccentricity is less than $0.5 \%$.

The other option - symmetrical set-up, i.e. buffer and reflector are made from the same material - requires ultrasonic measurements from the top and bottom anvil. Only one transducer for each wave type is concentrically assembled at one anvil's rear side. The advantage of this set-up is the optimum interference between direct and reflected waves because the transducer receives the reflected and interfered waves without any angular loss. On the other hand, the symmetrical set-up results in additional energy losses due to non-optimum impedance contrasts between sample and buffer/reflector. For symmetrical set-ups we used platinum at both sides, which is an optimum reflector, but a poor buffer resulting in additional reflection losses, especially at the platinum- $\mathrm{NaCl}$ interface. In case of measurements at elevated temperatures, not performed in this study, only one of the "ultrasonic" anvils can be grounded. Even by using a dc-power supply small fluctuations of the current result in interference with the ultrasonic signals.

For generation and detection of the ultrasonic waves we used lithium niobate transducers, cold covered, overtone polished with a natural frequency of $33.3 \mathrm{MHz}$ and a diameter of $5 \mathrm{~mm}$. They were cemented at the polished rear anvil side using epoxy resin diluted by acetone to reduce its viscosity for minimizing the thickness of the glue film. This is of fundamental importance for the interferometric method to ensure rigid coupling to the anvil, because it requires a broadband characteristics of the transducer as a result of

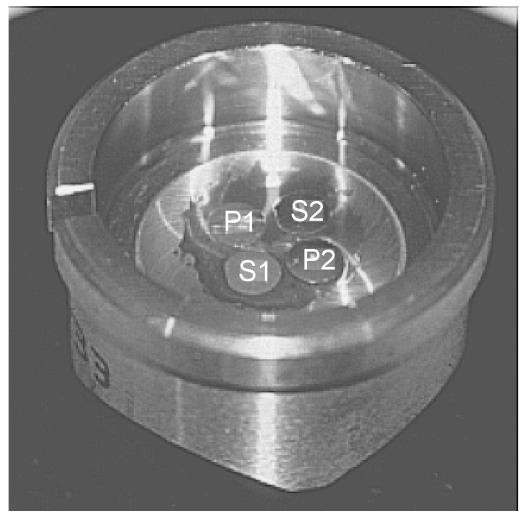

(b)

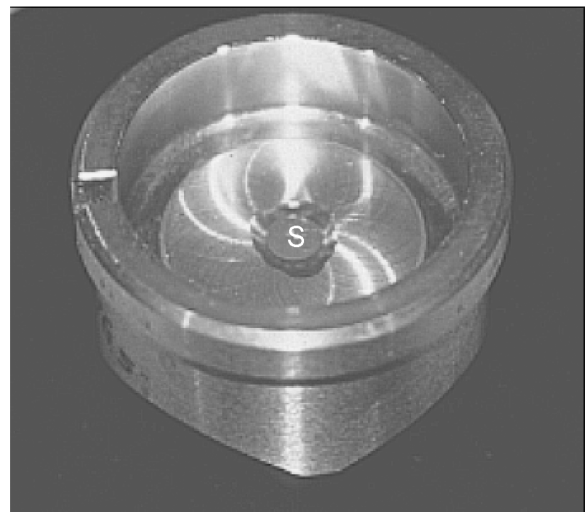

Figure 4. Transducer arrangements on the rear side of MAX80 anvils. (a) Two transducer couples for asymmetrical set-ups (b) Single transducer for symmetrical set-up. 
strong attenuation. The ultrasonic anvils are equipped with one $P$-wave or $S$-wave transducer, or with two couples of $P$-wave and $S$-wave transducers, respectively, depending on whether an asymmetrical set-up and two-transducer method, or a symmetrical set-up and single transducer method was used. First tests were performed with $3.5 \mathrm{~mm}$ truncation anvils made from cubic boron nitride $(\mathrm{cBN})$.

Figure 5 shows the electronic equipment for ultrasonic interferometry at MAX80. A PC-program controls the frequency sweep of the rf-generator by a frequency step of $100 \mathrm{kHz}$. An arbitrary waveform generator cuts wavelets (Shen et al., 1998) or double wavelets ( $\mathrm{Li}$ et al., 1998) with a duration of $20 \mathrm{~ns}$ to $4 \mu \mathrm{s}$ from the continuous sinusoidal signal of the rf-generator. The ultrasonic generator delivers the master trigger pulse and amplifies the received signal. For single transducer configurations, i.e. the transducer acts sequentially as generator and receiver of ultrasonic waves, a directional bridge is used to prevent the strong excitation wavelet from hitting the sensitive input of the receiving amplifiers. A power amplifier and pre-amplifier are used for samples with high damping or strong reflection losses at the interfaces. The multi-channel oscilloscope displays and digitizes the interference signals, finally stored on the PC's hard drive. The evaluation using an in-house computer program includes the selection and copying of the critical signal ranges, i.e. the buffer and sample reflections, their subtraction to isolate the interference between the signals, digital filtering, displaying the resulting periodic energy levels (constructive and destructive interferences) as a function of frequency, and finally displaying the resulting travel-time curves as a function of frequency as well (Mueller et al., 2003). The determined two-way travel time or its multiple inside the sample is represented by the bold straight line between the curves of opposite curvature in Figure 6.

\subsection{Determination of sample length}

The result of ultrasonic interferometric measurements is an equidistant sequence of critical frequencies for constructive and destructive interference of the reflected waves from the plane-parallel surfaces of the sample rod. Unfortunately the interference pattern does not only depend on the material properties of the sample, but also on sample length. Due to the sharp interference pattern, the travel time is determined with high precision - better than $0.4 \%$ - and the accuracy of the velocity determination mainly depends on the precision of the length measurement (Li et al., 2001). In situ sample length measurement in multi-anvil devices is not trivial, as it cannot simply be derived from measurements of the advance of anvils. Therefore, sample deformation models, derived from direct length measurements prior and after the experiment (Knoche et al., 1997, 1998) are common usage, or it is assumed that the sample deforms purely elastically. The so-called Cook's method (Cook, 1957) calculates the in situ sample length from the compressibility derived from measured elastic wave velocities. Our measurements with different samples in a variety of configurations showed that this assumption is only valid, if the sample is the strongest part of the buffer-sample-reflector combination. Knoche et al. (1997, 1998) had a hot isostatically pressed forsterite sample between two platinum buffer rods. Consequently, the condition mentioned can be a good approximation for high-strength samples, as it was also the case in our experiments with San Carlos olivine, anorthite, clinoenstatite, and 


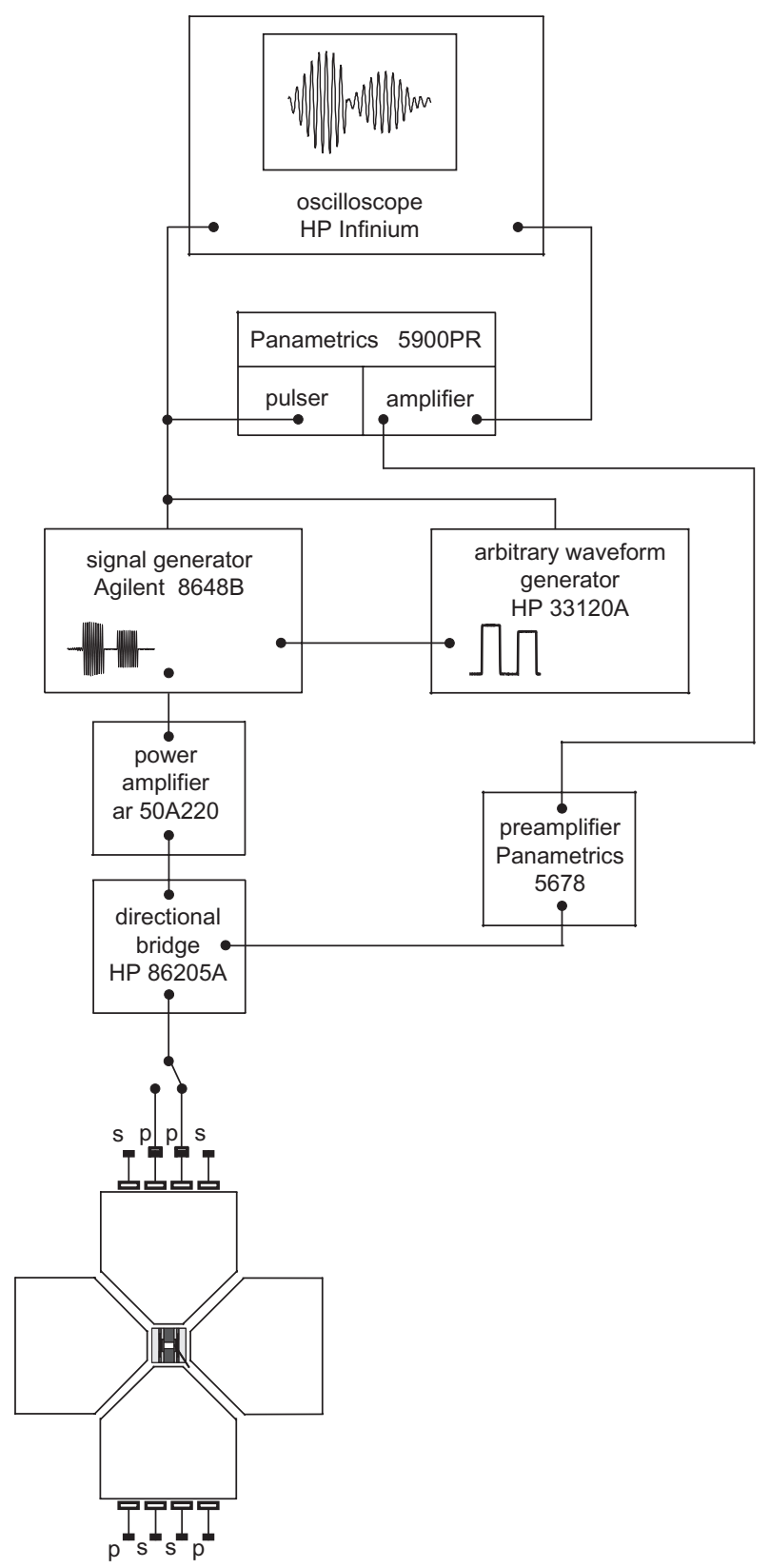

Figure 5. Electronic equipment for ultrasonic interferometry at MAX80. Rectangular pulses made by an arbitrary waveform generator gate a signal generator resulting in rf pulses or double-pulses. A directional bridge prevents the power burst from hitting the sensitive pre-amplifier. The oscilloscope displays and digitizes the received ultrasonic signals. Amplifiers and an integrated trigger source (5900PR) were used. Transducers were installed on modified top and bottom anvils of MAX80. A computer-controlled switch selects the active transducer or transducer pair. 


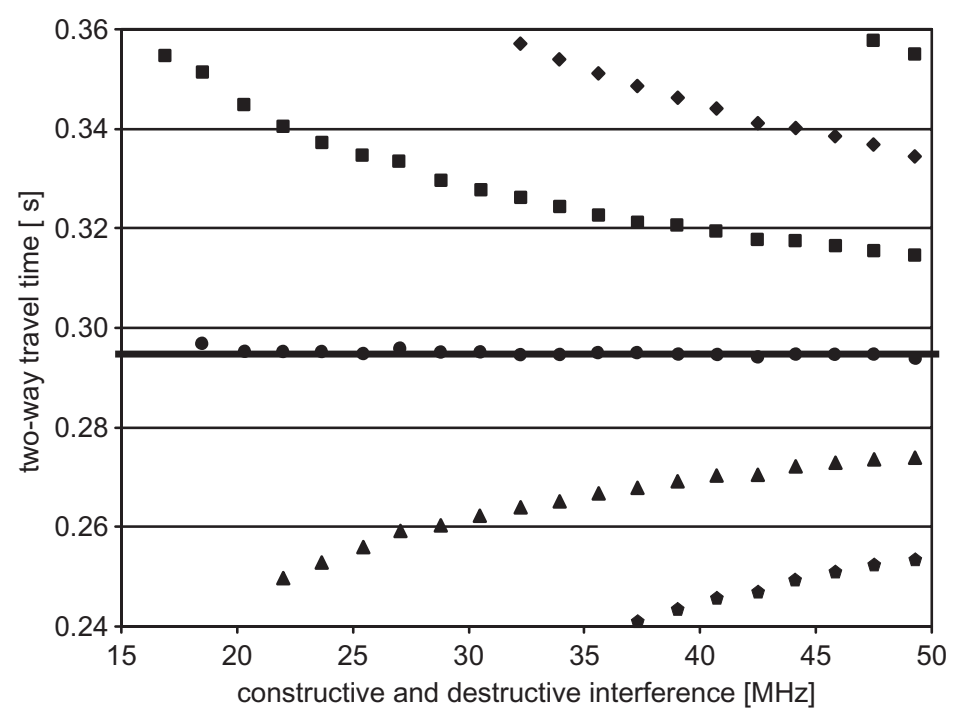

Figure 6. Typical resultant travel-time determination using interference pattern. Travel-time curves are plotted as a function of frequency at $7.71 \mathrm{GPa}$. Each point represents a frequency for constructive or destructive interference, and hence can be considered as an independent travel-time determination. The symbols fitted by the horizontal line represent the revealed travel time. The upper and lower curves represent neighboring fringes of interference pattern.

Q1 quartz (see Mueller et al. (2004), page Xxx, this volume), but not if a ductile sample is taken into account.

\subsubsection{XRD-scanning}

Contrary to experiments with high-strength samples, a NaCl-specimen between $\mathrm{Al}_{2} \mathrm{O}_{3}$ ceramics or iron and platinum buffers is the most ductile part and will accommodate large parts of the total deformation (Mueller et al., 2003). Because sodium chloride deforms as a combination of ductile and elastic behavior, simple deformation models are not useful and measurements under in situ conditions are necessary. An advantage of ultrasonic measurements at a radiation source is, that the sample length can be determined independently from the ultrasonic experiments. The first option is to scan the buffersample-reflector combination stepwise, crossing both the interfaces and determine the sample length by evaluating the in situ XRD-spectra (Fig. 7). The circles represent the X-ray beam radius of about $50 \mu \mathrm{m}$. The XRD-spectra close to the interface are a superposition of two spectra, because the X-ray beam penetrates both materials, i.e. Pt and $\mathrm{NaCl}$, to some degree. The whole press (including the multi-anvil device) can be lifted by stepper motors with an accuracy of $1 \mu \mathrm{m}$. By calculating the interface from the last and first pure spectrum the sample length can be determined much more precisely than the beam diameter is, that is, an accuracy of 5-10 $\mu \mathrm{m}$. An advantage of this method is, it requires no additional equipment and results in sufficient accuracy. The drawback is, it is highly time-consuming, about 20 min using the lowest step rate. 


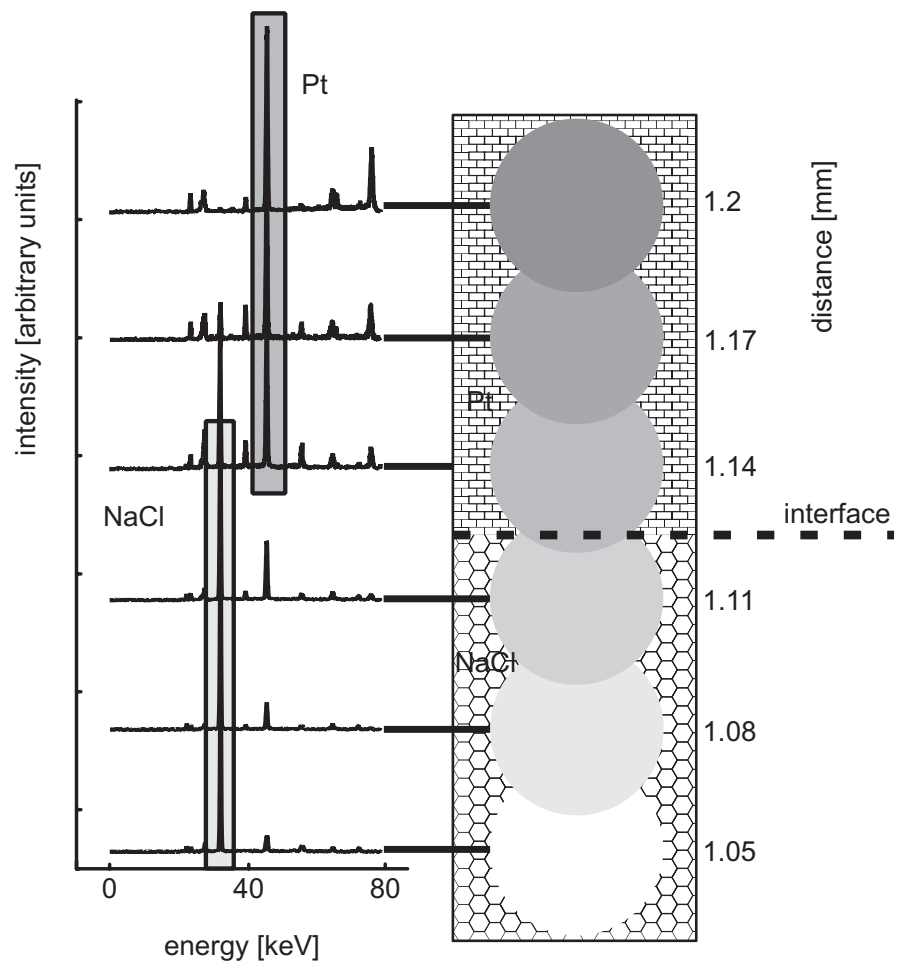

Figure 7. Determination of sample length under in situ conditions by XRD-scanning. The position of the interface is calculated as half the distance between the last and first appearance of pure XRD-spectra.

\subsubsection{X-radiography}

Li et al. (2001) used X-radiography to measure the sample length in multi-anvil devices under in situ conditions, after the method was established in the 1990s by other authors to observe under high-pressure conditions falling spheres in melts for viscosity measurements. At the latest when we introduced an ultrasonic data transfer function technique at Q1 MAX80 (see Mueller et al. (2004), page xxx, this volume) the XRD-scanning method was no longer adequate as the only available length measurement technique. A digital ultrasonic sweep for $v_{\mathrm{p}}$ and $v_{\mathrm{s}}$ lasts about $90 \mathrm{~min}$. Consequently, a duration of about $20 \mathrm{~min}$ for a XRD-scan of both interfaces was acceptable. But if the recording of two data transfer functions representing the whole $v_{\mathrm{p}}$ - and $v_{\mathrm{s}}$-data requires only some seconds, the length measurement becomes the limiting factor.

As the first step to establish a X-radiography system the fixed double-slits unit of MAX80 was exchanged by an adjustable slits system. We used a four-blade high-precision slits system of ADC (Fig. 8) equipped with four independent stepper motors including all the control electronics onboard. The maximum slits opening is $1 \mathrm{in}$. The motion repeatability is $1 \mu \mathrm{m}$ with a motion resolution of $0.4 \mu \mathrm{m}$. The MS Windows compatible IMS terminal software allows to control the slits system simply by the PC, already 


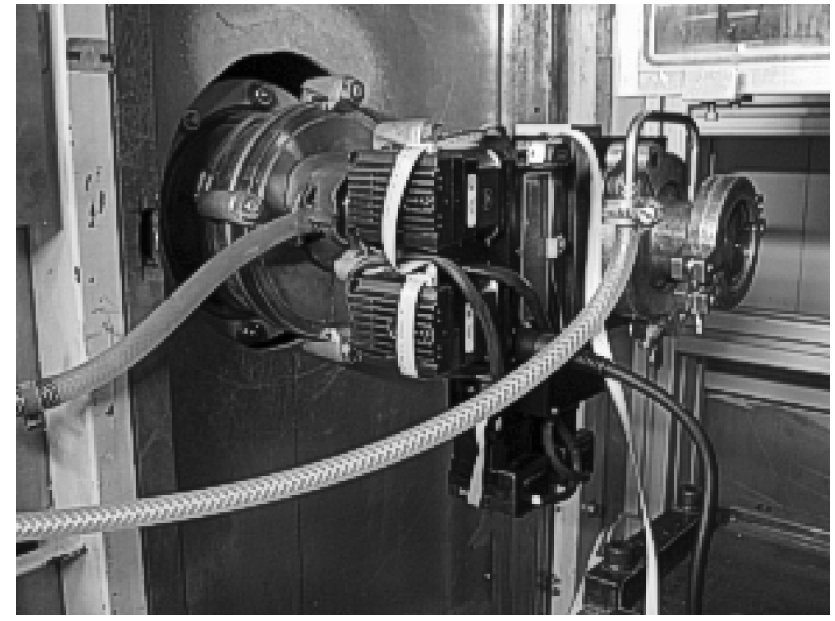

Figure 8. High-precision four-blade slits system.

installed for the ultrasonic measurements. Because the four blades can be moved independently from each other the slits system is able to define the X-ray beam position and size. Differently from the original state the X-ray beam position can be controlled by the slits and the positioning table of MAX80 now. For X-radiography the blades are opened so far, that the X-ray beam covers the whole sample length including a part of the adjacent buffer and reflector rods. Using tungsten carbide anvils absorbing the synchrotron radiation (intense X-rays) the maximum vertical opening of the beam is adapted to the maximum available gap between the lateral anvils, of about $1.5 \mathrm{~mm}$ at normal pressure and less than $0.5 \mathrm{~mm}$ at maximum conditions. To limit the scattered radiation inside the hutch, the slits is only opened to the size necessary for the sample length measurement.

First of all the X-radiography system (Fig. 9) consists of an $0.1 \mathrm{~mm}$ thick Ce:YAGcrystal (by courtesy of IKZ) of $15 \mathrm{~mm}$ diameter in an adjustable aluminum mounting. It partially converts the X-ray shadow graph after passing through the set-up by

4-blade high precision slits system

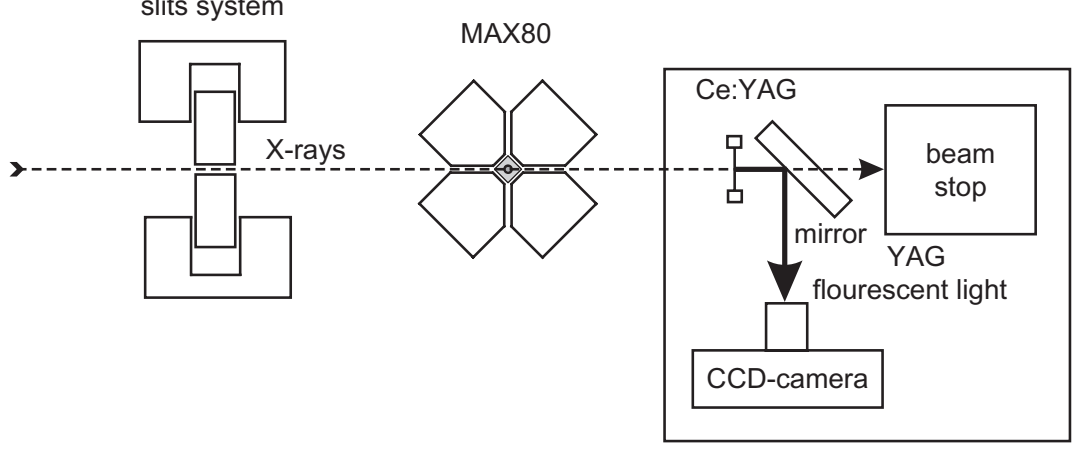

Figure 9. Scheme of X-radiography at MAX80. 


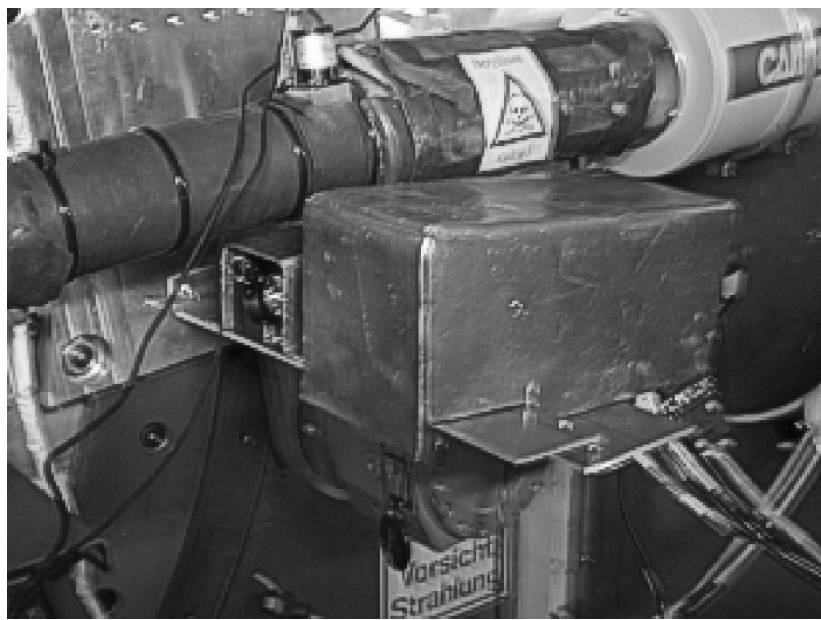

Figure 10. X-radiography system without camera inside PB-shielding below the XRD-detector.

fluorescence to an optical image of about $540 \mathrm{~nm}$ wavelength (light green), which is redirected to a CCD-camera by an aluminum-coated mirror. A beam-stop behind the mirror absorbs the non-converted X-rays. The Ce:YAG-crystal should be made as thin as possible to limit the warming up by X-ray absorption and to keep the optical image as sharp as possible, because the fluorescence creates optical images at all atomic planes inside the crystal. Extensive use of aluminum for X-ray exposed components is recommended to limit the warming of the parts by absorption. The decoupling of the optical image from the X-ray shadow graph by the mirror is necessary to prevent the $\mathrm{CCD}$-camera from direct X-ray flux. The whole system is covered by a $2.5 \mathrm{~mm}$ thick $\mathrm{Pb}$ casing for shielding from scattered radiation inside the hutch (Fig. 10).

For taking images optimum for the following evaluation each shadow graph was recorded with three different exposure times, differing from each other by the fourfold exposure. The automatic exposure control failed because of the high-intensity contrasts of the images. The evaluation of the shadow graphs is performed by densitometry profiling, i.e. the image processing software analyzes the brightness of the image along a pre-defined line. Figure 11 shows the shadow graph and the related image processing result for a $\mathrm{NaCl}$-sample at $5 \mathrm{GPa}$ pressure in linear and logarithmic scale. At the optimum exposition time the low-dense $\mathrm{NaCl}$ is displayed as pure white. The sample length, i.e. the number of zero density pixels at the central part of the image, is 149 pixels. Because of the small, but existing divergence of the X-rays, the shadow graphs and the sample have not necessarily the same size. Therefore, the shadow graphs are calibrated, before the high-pressure run starts, because at this time the sample length is exactly known from the preparation. From this calibration we know that the 149 pixels, displayed in Figure 11 represent a sample length of $1.94 \mathrm{~mm}$. This means the accuracy is 1 pixel, i.e. $0.013 \mathrm{~mm}$.

What are the accuracy limits for X-radiography? On principle the wavelength of light, i.e. about $0.5 \mu \mathrm{m}$, limits the resolution. But in reality it gets worse, because the aperture of the objective is less than 0.5 . To keep the camera outside the intensive X-rays, the working distance must be about $40 \mathrm{~mm}$, very large, i.e. very disadvantageous for a micro objective. 
(a)

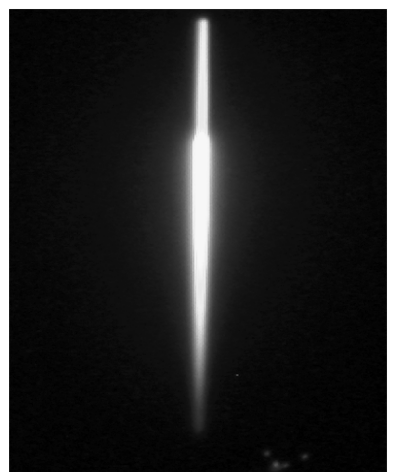

linear density

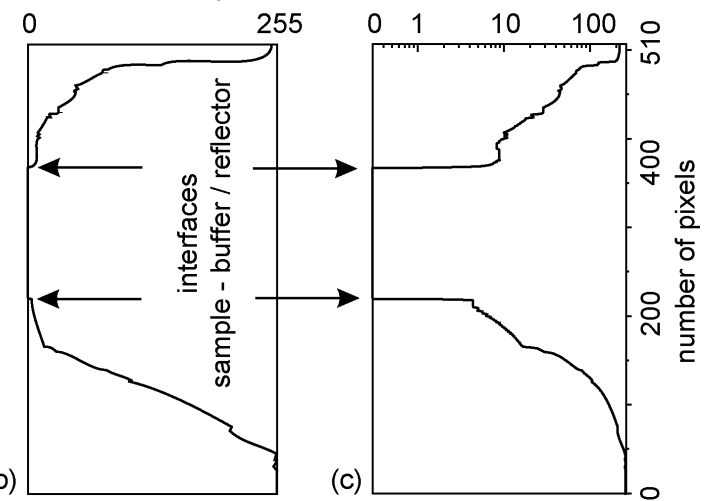

Figure 11. X-ray shadow graph (a) and its evaluation by density analysis in linear (b) and logarithmic scale (c).

This limits the practical accuracy to about $1 \mu \mathrm{m}$, which is a half order of magnitude better than X-ray scanning at the minimum. First results with a conventional consumer 5 megapixels color camera with a minimum working distance of $70 \mathrm{~mm}$ demonstrate the potential of the used set-up and confirm the results of XRD-scanning. Because the image processing only uses the density of the image, first the color image is converted to a gray scale one. Therefore, in the next experiments a 6 megapixels black and white CCD-camera will be used at a working distance of $40 \mathrm{~mm}$ to guarantee a $1 \mu \mathrm{m}$ resolution.

\subsection{Experimental procedures}

Three experiments were included in the evaluation. Run 2.2 is one of the first ultrasonic experiments at all performed simultaneously with synchrotron XRD-maintenance in MAX80. Due to the similarity between the set-up of run 3.10 and 2.2, the sample deformation measured by synchrotron radiation during run 3.10 was used for both experiments. Set-up 2.2 had a buffer made of glass ceramics; set-up 3.10 had an iron buffer. Both asymmetrical set-ups had platinum reflectors. To make the pressure per load and deformation results comparable to other experiments the set-ups had a stepped graphite heater which was not in use during these experiments.

Run 3.27 used six cBN anvils with $3.5 \mathrm{~mm}$ truncation to increase the maximum pressure. Because $\mathrm{cBN}$ is an electrical insulator the rear side of the top and bottom anvil got a goldplatinum electrode for the transducers by sputtering. The top anvil was equipped with pairs of $p$ - and $s$-wave transducers. In addition to that, the bottom anvil was equipped with a single $p$-wave transducer to compare the results of both configurations (see Figure 4 ). Due to electrical contact failure at the bottom piston only the symmetrical set-up with two platinum buffers could be used. The much smaller anvil truncation require boron-epoxy cubes of $5.5 \mathrm{~mm}$ length. To enlarge the reflection surface, i.e. to have a higher sample diameter a special set-up was designed without heater and insulator tube (Fig. 3). The experiment showed that the friction between anvil's surface and gaskets was much higher than using tungsten carbide anvils resulting in a maximum pressure of $7.71 \mathrm{GPa}$. 


\subsection{Gasket insets - anvil support}

In normal use MAX80 forms the gaskets between the anvils from the boron epoxy cube's material during the runs. This allows a simple and rapid sample change, ensures low X-ray energy loss by any additional materials and results in a good "high-pressure efficiency", i.e. for a given load the pressures are relatively high, because the small gaskets formed by the cubes reduce the additional surface and hence, the "unproductive" part of the load. On the other hand, a better lateral anvil support by an additional gasket results in a more homogeneous stress distribution inside the anvils leading to a higher maximum force to the sample cube and consequently higher pressures inside the set-up. Prefabricated gasket insets, normal for all double-stage multi-anvil devices, are a way for lateral anvil support at the expense of a lower pressure efficiency. For first tests we used gasket strips made from Klinger SIL C-4400 (Fig. 12), an industrial sealing material made from NBR tied $p$-aramide fibers for tungsten carbide and $\mathrm{cBN}$ anvils. The post-experimental optical inspection of the tungsten carbide anvils showed that the material starts to flow at the corners of the front face without any failure of the anvil. Because the gap width between the anvils was larger at elevated pressures the X-ray intensity was higher and the adjustment of the ray was easier. In other words, due to the reduced pressure efficiency the maximum pressure could be enhanced and the XRD measurements could be improved. For cBN anvils the friction between the gasket material and the anvils was too high, resulting in a stick-slip behavior and material failure. Material and shape of the gasket insets will further be optimized for future experiments.

The first experiments with tungsten carbide anvils showed $\approx 25 \%$ higher maximum pressures compared to the standard MAX80 configuration because of increased lateral

\section{Q3}

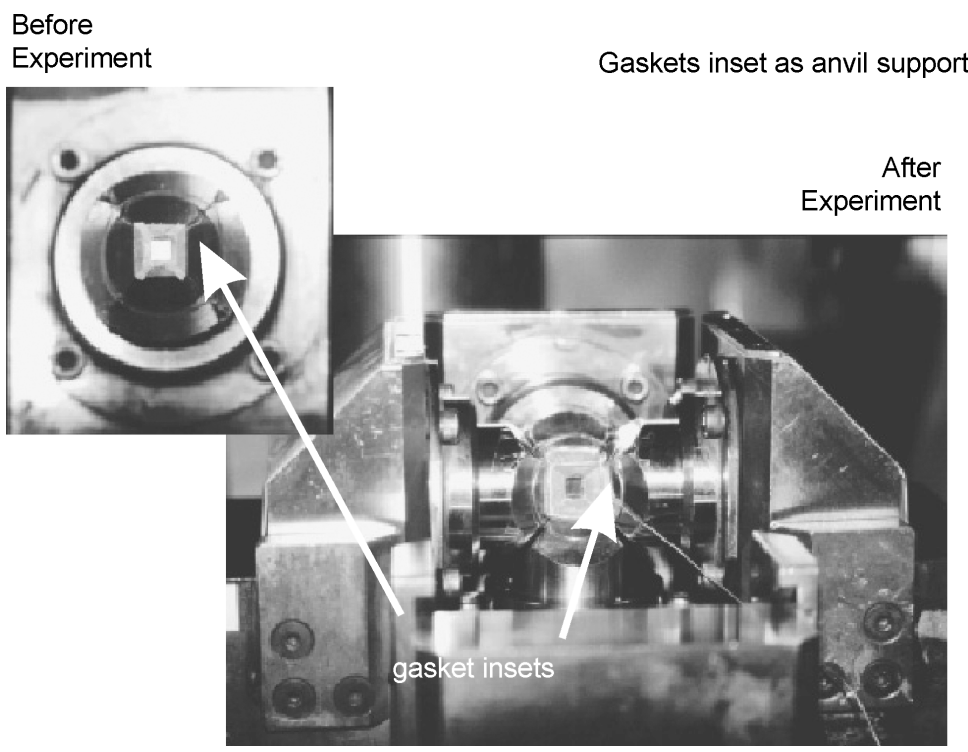

Figure 12. Prefabricated gasket insets prior and after the high-pressure run. 
anvil support, reduced number of blow-outs, higher X-ray intensity, and a reduced probability of thermocouple cut-off during the experiments.

\subsection{Samples}

Sodium chloride powder of $99.5 \%$ purity (analytical grade by Merck) was used as starting material. The mean grain size was $50 \mu \mathrm{m}$. The powder was pressed to a sample cylinder of $10 \mathrm{~mm}$ diameter and a length of $20 \mathrm{~mm}$ using a load of 6 tons resulting in an effective pressure of $0.25-0.3 \mathrm{GPa}$. The millimeter sized samples (diameter 2.4 and $1.6 \mathrm{~mm}$ length for $6 \mathrm{~mm}$ anvil truncation, and diameter 3.1 and $1.1 \mathrm{~mm}$ length for $3.5 \mathrm{~mm}$ anvil truncation) for the high-pressure experiments were shaped with a high-precision $( \pm 0.5 \mu \mathrm{m})$ cylindrical grinding machine and polished at the plane-parallel faces of the sample rod.

\section{Results and discussion}

The digitized interferometric signals stored on a PC's hard drive were processed using an in-house program. The resulting sequence of maxima and minima represents the frequencies for constructive and destructive interference. Picking all available maxima and minima as a function of frequency $\nu$ allows the determination of the travel time $\tau$ inside the sample as the regression result for the horizontal point sequence between the curves of opposite curvature (Fig. 6). The curvature is the result of an inappropriate use of the order of interference $n$ according to $\tau=n 1 / \nu$.

The calculation of wave velocities requires the sample length as a function of pressure. Consequently, the precise measurement of sample deformation during the experiment is essential for the accuracy of the whole method, because for higher degrees of deformation this contribution to the critical frequency interval can be higher than that of the variation of sample's elastic properties. Figure 13 is the plot of $v_{\mathrm{p}}$ and $v_{\mathrm{s}}$ for the three experimental runs. The results for our runs are in agreement with previous results published by Frankel et al. (1976) within the limit of experimental errors $(\sim 1.5 \%)$.

The velocities of run 3.27 are located between the values of the other two experiments and are used as average value. This run reaches the highest pressure and was used for further modeling.

The measured elastic wave velocities $v_{\mathrm{p}}$ (compressional wave) and $v_{\mathrm{s}}$ (shear wave) were used to calculate the adiabatic bulk modulus $K_{\mathrm{S}}$ and the corresponding compressibility $\kappa_{\mathrm{S}}$.

$$
K_{\mathrm{S}}=\rho\left(v_{\mathrm{p}}^{2}-\frac{4}{3} v_{\mathrm{s}}^{2}\right)
$$

and

$$
\kappa=\frac{1}{K_{\mathrm{S}}}
$$

This calculation requires the density $\rho$ of the sample as a function of pressure which is directly obtained by XRD measurements. In situ sample length measurement is the 

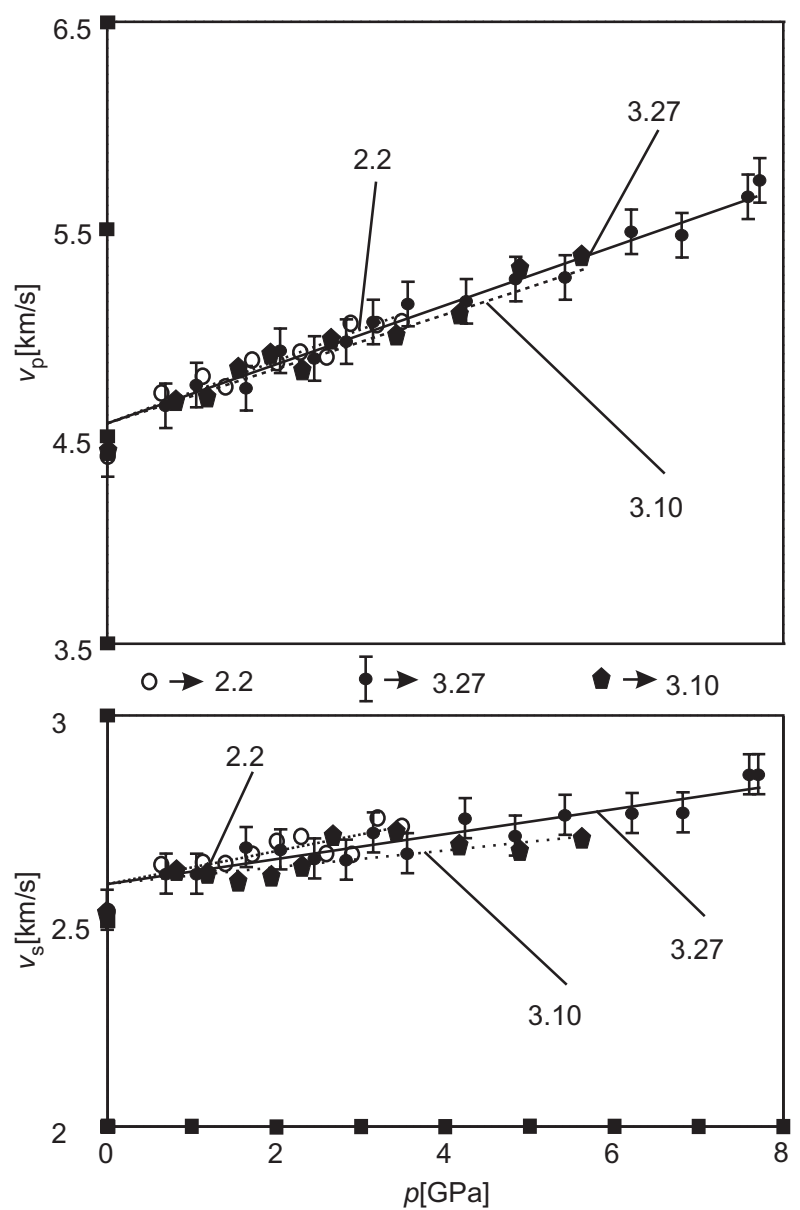

Figure 13. Elastic wave velocities $v_{\mathrm{p}}$ and $v_{\mathrm{s}}$ of polycrystalline $\mathrm{NaCl}$ at high pressure. Runs 2.2 and 3.10 use $8 \mathrm{~mm}$ set-ups for $6 \mathrm{~mm}$ anvil truncation; run 3.27 uses a $5.5 \mathrm{~mm}$ set-up for $3.5 \mathrm{~mm}$ anvil truncation.

important basis, but in addition to that the study of the lateral deformation is also necessary. That becomes the more important, the less hydrostatic the pressure and the more ductile the sample is. Therefore, a user of any multi-anvil device has to take care of set-up deformation. Different methods exist to meet the demands.

The general form of the EoS is:

$$
P(V, T)=P_{1}(V)+P_{\mathrm{TH}}(V, T)
$$

where $P_{1}$ refers to the isothermal EoS, and $P_{\mathrm{TH}}$ refers to the thermal pressure. For small compressions, the isothermal bulk modulus $K_{\mathrm{T}}$ can be approximated by:

$$
K_{T}=-V\left(\frac{\partial P}{\partial V}\right)_{T}=K_{0}+P K_{0}^{\prime}+\frac{P^{2} K_{0}^{\prime \prime}}{2}+\cdots
$$


Here $K_{0}, K_{0}{ }^{\prime}$, and $K_{0}{ }^{\prime \prime}$ are zero-pressure values of $K$ and its first and second pressure $P$ derivatives, at constant temperature. The first two terms usually suffice to represent ultrasonic measurements, but $K_{0}^{\prime \prime}$ appears to be negative and of a magnitude such that a quadratic in $P$ leads to $K=0$ (Birch, 1978). Therefore, only $K_{0}$ and $K_{0}{ }^{\prime}$ are used. Using published data for $K, K^{\prime}$ and density at normal pressure $\rho_{0}$ (Birch, 1978, 1986; Holland and Ahrens, 1998) the density at given pressure can be calculated:

$$
\frac{V}{V_{0}}=1-\kappa P
$$

and

$$
\rho_{\mathrm{P}}=\frac{V_{0}}{V} \rho_{0}
$$

Another widely used approach is measuring and deriving the deformation of the sample from the ultrasonic experiment itself, called Cook's method (Cook, 1957; Kung et al., 2001a,b).

$$
\begin{aligned}
& S=1+\frac{1+\alpha \gamma T}{3 h_{0}} \int_{0}^{P} \frac{\mathrm{d} P}{\left(\frac{1}{t_{\mathrm{p}}^{2}}-\frac{4}{3} \frac{1}{t_{\mathrm{s}}^{2}}\right)} \\
& h_{0}=4 \rho_{0} l_{0}^{2}
\end{aligned}
$$

where $S$ is linear compression, $\alpha$ linear thermal expansion coefficient, $\gamma$ thermodynamical Grüneisen parameter, $T$ absolute temperature, $P$ pressure, $\rho_{0}$ density at zero-pressure, $l_{0}$ sample length at zero-pressure, $t_{\mathrm{p}}$ travel time of compressional waves along the sample, and $t_{\mathrm{s}}$ is travel time of shear waves along the sample.

But this is only valid for

$$
\frac{\rho}{\rho_{0}}=\left(\frac{l_{0}}{l}\right)^{3}=S^{3}
$$

which means the deformation is purely hydrostatic, i.e. uniform in all directions of space. However, our post-experimental examination of the set-up showed that this boundary condition is not achieved for our set-up and non-encapsulated $\mathrm{NaCl}$-samples, because the sample is the most ductile part of the set-up. As a consequence of the gasket formation there is a reel-shaped deformation of the sample, i.e. the length decreases, the diameter at half the sample length slightly decreases or keeps constant, but the diameter at the front faces increases. Some minor parts of the material can be even squeezed out there. Therefore, we used a more generalized equation published by Frankel et al. (1976). For a material whose EoS is unknown, Katz and Ahrens (1963) showed that an EoS can be solved for by assuming that the geometry of the specimen changes under pressure such that

$$
\rho=\rho_{0} X^{n}
$$


where

$$
X=\frac{l_{0}}{l}
$$

where $X$ is geometry characteristics.

The parameter $n$ is any positive number and is assumed to be independent of pressure. The change in the specimen density and thickness can be determined from the data as follows:

$$
X^{n-2}=1+\left(\frac{n-2}{n}\right) \frac{1}{4 l_{0}^{2} \rho_{0}} \int_{0}^{P} Y \mathrm{~d} P
$$

For $n \neq 2$, and

$$
X=\exp \left[\frac{1}{8 l_{0}^{2} \rho_{0}} \int_{0}^{P} Y \mathrm{~d} P\right]
$$

For $n=2$, where

$$
\begin{aligned}
& Y=\frac{1+\Delta}{\Delta f_{\mathrm{p}}^{2}-\frac{4}{3} \Delta f_{\mathrm{s}}^{2}} \\
& \Delta f_{\mathrm{p}}=\frac{v_{\mathrm{p}}}{2 l} \\
& \Delta f_{\mathrm{s}}=\frac{v_{\mathrm{s}}}{2 l} \\
& \Delta=\frac{9 \alpha^{2} T K_{\mathrm{S}}}{\rho C_{\mathrm{p}}}
\end{aligned}
$$

where $\Delta f_{\mathrm{p}}$ is frequency interval between two critical frequencies for compressional waves, $\Delta f_{\mathrm{s}}$ frequency interval between two critical frequencies for shear waves, and $C_{\mathrm{p}}$ is specific heat at constant pressure.

If the forces acting upon a specimen are perfectly balanced, such as they are in a liquid pressure transmitting medium, the parameter $n$ in Eq. (10) is equal to 3.0. All strains are due to hydrostatic stresses. The assumption of hydrostatic compression led Ahrens and Katz (1962) to use an expression as Cook's method identical to Eq. (12) with $n=3$. If the deformation of the specimen is piston-like, i.e. the side walls are rigid and only the thickness changes, then the value of $n$ is 1.0. If the sidewalls - as in our experiments - are rigid or more easily deformable than the buffers in axial direction, $n \geq 1.0$. We found $n=0.622$ for run 3.27 .

A third possibility to determine density as a function of pressure is an iterative numerical approach. The calculation of the adiabatic bulk modulus at the first pressure step starts with the assumption $\rho=\rho_{0}$, i.e. the density do not change within this small pressureinterval. The resulting compressibility is used to calculate the increased density at this 
pressure, which is used for the next calculation cycle. The result is very close to the data determined by using the EoS published by Ahrens and Katz (1962) and Birch, (1986).

The in situ density evaluation was performed while using unit cell parameters of $\mathrm{NaCl}$ derived by XRD.

$K_{0}$ - and $K_{T}^{\prime}$ - values published by Birch (1986) were used to calculate the isothermal bulk modulus $K_{T}$ and the corresponding compressibility $\kappa_{T}$ using Eqs. (2) and (4). The $V / V_{0}$ values published by Bridgman (1940) were also used to calculate the isothermal compressibility. Both values agree very well (Fig. 14). The difference between the adiabatic $\left(K_{\mathrm{S}}\right)$ and isothermal $\left(K_{T}\right)$ bulk moduli is

$$
K_{\mathrm{S}}=K_{T}(1+\alpha \gamma T)
$$

and $\alpha \gamma T \approx 0.01$ at room temperature (Kung and Rigden, 1999) was taken into account.

The detailed comparison of the data showed minor differences. The ultrasonic curves cross the static compression graphs twice. At $\approx 1.2 \mathrm{GPa}$ the compressibility graph derived from the EoS-fitted ultrasonic data intersects the static compression graph first. At ambient conditions the static compressibility is $7 \%$ higher than the dynamical compressibility derived from ultrasonic measurements. This seems to be the result of non-intrinsic compression, e.g. due to a closure of micro-cracks at the early compression stage in static compression experiments.

Between 2 and $4 \mathrm{GPa}$ the graphs are nearly parallel with up to $3 \%$ higher compressibility derived from ultrasonic measurements. The high-pressure intersection is located at 5.3 GPa. At higher pressures the difference seems to increase. At our maximum pressure of $7.71 \mathrm{GPa}$ the static compressibility is again $6.6 \%$ higher than the presented value. This may lead to significant errors for the pressure standard at higher pressure.

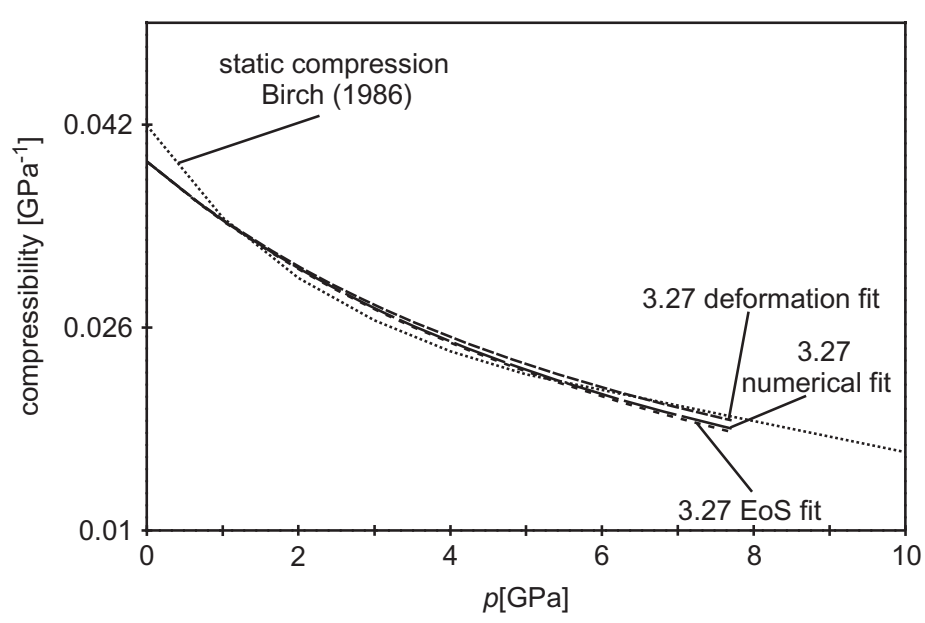

Figure 14. Compressibility of $\mathrm{NaCl}$ measured by ultrasonic interferometry and static compression: The calculation of compressibility from elastic wave velocities require the density as a function of pressure. The in situ density was determined by analyzing the sample deformation (deformation fit), using published EoS (EoS-fit) and successive approximation. The $X$-axis is related to the Decker (1971) pressure scale. 
Table 1. Polynomial fit coefficients for the compressibility of $\mathrm{NaCl}$ measured by ultrasonic interferometry and static compression.

\begin{tabular}{|c|c|c|c|c|}
\hline $\begin{array}{l}\text { Polynomial fit } \\
\text { coefficients } \\
\text { (Eq. (19)) }\end{array}$ & $\begin{array}{l}\text { Static } \\
\text { compression }^{\mathrm{a}}\end{array}$ & $\begin{array}{l}\text { Ultrasonics, } \\
\rho \text { from } \mathrm{EoS}^{\mathrm{b}}\end{array}$ & $\begin{array}{l}\text { Ultrasonics, } \\
\rho \text { from } \\
\text { deformation }^{\mathrm{c}}\end{array}$ & $\begin{array}{l}\text { Ultrasonics, } \\
\rho \text { from num. } \\
\text { approach }^{\mathrm{d}}\end{array}$ \\
\hline$\pi$ & 0.04191 & 0.03907 & 0.03907 & 0.0 \\
\hline$B_{1}$ & -0.0088 & -0.00523 & -0.00514 & -0.00514 \\
\hline$B_{2}$ & 0.0018 & $6.51178 \times 10^{-4}$ & $6.50735 \times 10^{-4}$ & $6.00421 \times 10^{-4}$ \\
\hline$B_{3}$ & $-2.43008 \times 10^{-4}$ & $-1.09931 \times 10^{-4}$ & $-1.02209 \times 10^{-4}$ & $-9.2428 \times 10^{-5}$ \\
\hline$B_{4}$ & $1.93729 \times 10^{-5}$ & $2.19162 \times 10^{-5}$ & $1.86429 \times 10^{-5}$ & $1.83552 \times 10^{-5}$ \\
\hline$B_{5}$ & $-8.72196 \times 10^{-7}$ & $-3.17726 \times 10^{-6}$ & $-2.56361 \times 10^{-6}$ & $-2.71682 \times 10^{-6}$ \\
\hline
\end{tabular}

${ }^{\text {a }}$ Compressibility measured by static compression (Birch, 1986).

b Compressibility measured by ultrasonic interferometry (this work), density $\rho$ was derived from EoS (Birch, 1986).

${ }^{\mathrm{c}}$ Compressibility measured by ultrasonic interferometry (this work), density $\rho$ was derived from sample deformation (Ahrens and Katz, 1962).

${ }^{\mathrm{d}}$ Compressibility measured by ultrasonic interferometry (this work), density $\rho$ was calculated by an iterative numerical approach.

In terms of pressure measurement the compressibility calculated from ultrasonic data indicate at $3 \mathrm{GPa}$ about $0.25 \mathrm{GPa}$ higher pressures than derived from static compression data by Bridgman (1940). The ultrasonic data are related to Decker (1971) pressure scale.

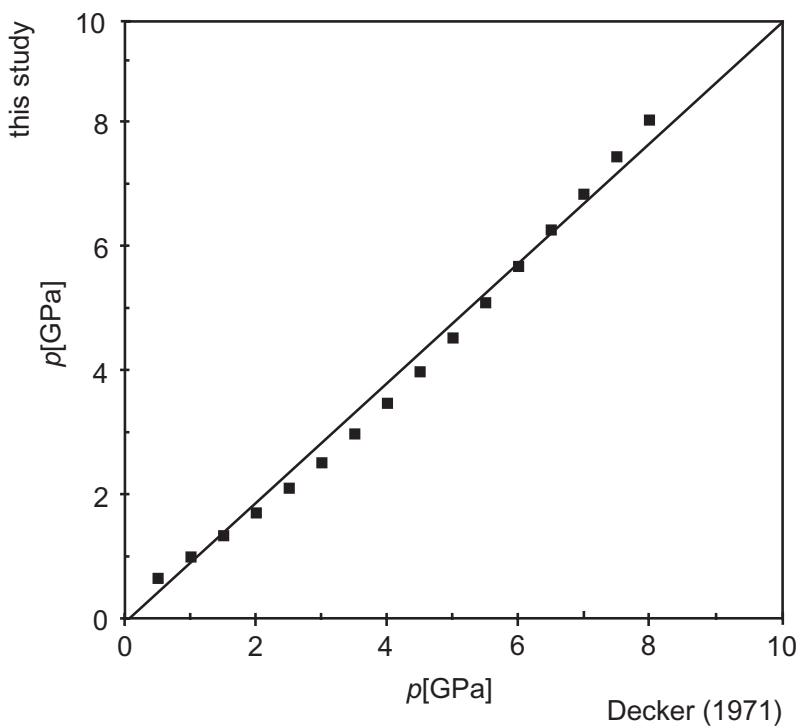

Figure 15. Pressure measured by ultrasonic interferometry in this study vs. Decker (1971) pressure scale related to the EoS by Birch (1986). 
The graphs for all calculated $\mathrm{NaCl}$ compressibilities, i.e. derived from static compression and from ultrasonic measurements using the EoS, the empirical deformation model, and the numerical approach, were polynomial fitted up to the power of 5, which is required for the range between 1 and $5 \mathrm{GPa}$. Table 1 presents the coefficients of this fits according to Eq. (19).

$$
K_{T}=A+B_{1} P+B_{2} P^{2}+B_{3} P^{3}+B_{4} P^{4}+B_{5} P^{5}
$$

Figure 15 shows the relation between the Decker (1971) pressure scale and the pressure derived from the ultrasonic measurements of this study using the Eos by Birch (1986). The data were also polynomial fitted up to the power of 5:

$$
\begin{aligned}
p_{\text {us }}= & 0.34611+0.6807 p_{\mathrm{De}}+0.01921 p_{\mathrm{De}}^{2}+0.00246 p_{\mathrm{De}}^{3}+8.4777 \times 10^{-4} p_{\mathrm{De}}^{4} \\
& +5.75971 \times 10^{-5} p_{\mathrm{De}}^{5}
\end{aligned}
$$

where $p_{\mathrm{us}}$ is pressure derived from ultrasonic measurements of this study and $p_{\mathrm{De}}$ is pressure according to Decker (1971).

\section{Conclusions}

The results demonstrate the ability to measure the pressure inside of multi-anvil pressure cells standard-free by ultrasonic interferometry. The synchrotron radiation is used to measure the pressure by XRD-techniques using EoS after Decker (1971). The synchrotron radiation is also used for precise in situ sample length and density determination required for the ultrasonic method. Different ways of density determinations were used (using the EoS for $\mathrm{NaCl}$, published by Birch (1986), analyzing the deformation (Ahrens and Katz, 1962), and using an iterative numerical approach) and agreed within $<0.1 \%$. Ultrasonic pressure measurement will probably not substitute the XRD-determination completely, because of its higher technical expense, but might be important for a calibrant-free pressure scale determined at very high pressures. However, it seems to become a standard high-pressure method to determine elastic properties of polycrystalline samples parallel to the growing amount and quality of ultrasonic measurements on single crystals under experimental simulated Earth's mantle conditions.

\section{Acknowledgements}

We would like to express our special thanks to the editors J. Chen, Y. Wang, T. Duffy, G. Shen, and L. Dobrzhinetskaya for their initiative, patience, and guidance, as well as two unknown referees for their very constructive reviews. We are especially grateful to S. Ganschow for the Ce:YAG crystal, manufactured and put at our disposal by IKZ and J. Kulesza (ADC) for his untiring support. The authors thank M. Kreplin and G. Berger for fabricating tools and cell parts, C. Karger for her software contribution, and H. Witzki and W. Steiner for many initiatives and important technical contributions as well as all colleagues of the high-pressure mechanical workshop for their dedicated support. 


\section{References}

Ahrens, T.J., Katz, S., 1962. An ultrasonic interferometer for high-pressure research. J. Geophys. Res. 67, 2935-2944.

Bassett, W.A., Reichmann, H.-J., Angel, R.J., Spetzler, H., Smyth, J.R., 2000. New diamond anvil cells for gigahertz ultrasonic interferometry and X-ray diffraction. Am. Mineral. 85, 283-287.

Birch, F., 1978. Finite strain isotherm and velocities for single-crystal and polycrystalline $\mathrm{NaCl}$ at high pressures and 300 K. J. Geophys. Res. 83, 1257-1268.

Birch, F., 1986. Equation of state and thermodynamic parameters of $\mathrm{NaCl}$ to $300 \mathrm{kbar}$ in the hightemperature domain. J. Geophys. Res. 91, 4949-4954.

Q2 Boehler, R., Kennedy, G.C., 1980. Equation of state of sodium chloride up to $32 \mathrm{kbar}$ and $500^{\circ} \mathrm{C}$. J. Phys. Chem. Solids 41, 517-523.

Bridgman, P.W., 1940. Compressions to 50,000 kg/cm². Phys. Rev. 57, 237-239.

Brown, J.M., 1999. NaCl pressure standard. J. Appl. Phys. 86, 5801-5808.

Chen, J., Parise, J.B., Li, R., Weidner, D.J., Vaughan, M., 2000. The imaging plate system interfaced to the large-volume press at beamline X17B1 of the national synchrotron light source. In: Manghnani, M.H., Yagi, T. (Eds), Properties of Earth and Planetary Materials at High Pressure and Temperature, Geophysical Monograph 101. AGU, Washington, DC, pp. 139-144.

Cook, R.K., 1957. Variation of elastic constants and static strains with hydrostatic pressure: a method for calculation from ultrasonic measurements. J. Acoust. Soc. Am. 29, 445-449.

Decker, D.L., 1971. High-pressure equation of state for $\mathrm{NaCl}, \mathrm{KCl}$, and $\mathrm{CsCl}$. J. Appl. Phys. 42 , 3239-3244.

Frankel, J., Rich, F.J., Homan, C.G., 1976. Acoustic velocities in polycrystalline $\mathrm{NaCl}$ at $300 \mathrm{~K}$ measured at static pressures from 25 to 270 kbar. J. Geophys. Res. 81, 6357-6363.

Q2 Fritz, J.N., Marsh, S.P., Carter, W.J., Mcqueen, R.G., 1971. The Hugonoit equation of state of sodium chloride in the sodium chloride structure. In: Lloyd, E.C. (Ed.), Accurate Characterization of the High Pressure Environment, NBS Special Publication, Vol. 326, pp. 201-208.

Getting, I.C., 1998. The practical pressure scale: fixing fixed points and future prospects. Eos Trans., AGU 79, F830.

Holland, K.G., Ahrens, T.J., 1998. Properties of LiF and $\mathrm{Al}_{2} \mathrm{O}_{3}$ to $240 \mathrm{GPa}$ for metal shock temperature measurements. In: Manghnani, M.H., Yagi, T. (Eds), Properties of Earth and Planetary Materials at High Pressure and Temperature, Geophysical Monograph 101. AGU, Washington, DC, pp. 335-343.

Katz, S., Ahrens, T.J., 1963. Ultrasonic measurements of elastic properties of small specimens at high pressure. High Pressure Meas. Pap. 1962, 246-261.

Knoche, R., Webb, S.L., Rubie, D.C., 1997. Experimental determination of acoustic wave velocities at Earth mantle conditions using a multianvil press. Phys. Chem. Earth 22, 125-130.

Knoche, R., Webb, S.L., Rubie, D.C., 1998. Measurements of acoustic wave velocities at $P-T$ conditions of the Earth's mantle. In: Manghnani, M.H., Yagi, T. (Eds), Properties of Earth and Planetary Materials at High Pressure and Temperature, Geophysical Monograph 101. AGU, Washington, DC, pp. 119-128.

Kung, J., Rigden, S., 1999. Oxide perovskites: pressure derivatives of the bulk and shear moduli. Phys. Chem. Miner. 26, 234-241.

Kung, J., Gwanmesia, G.D., Liu, J., Li, B., Liebermann, R.C., 2000. PV3T experiments: simultaneous measurement of sound velocities $\left(V_{\mathrm{p}}\right.$ and $\left.V_{\mathrm{s}}\right)$ and sample volume $(V)$ of polycrystalline specimens of mantle minerals at high pressures $(P)$ and temperatures $(T)$. Eos Trans., AGU 81, 48, F1151.

Kung, J., Angel, R.J., Ross, N.L., 2001a. Elasticity of $\mathrm{CaSnO}_{3}$ perovskite. Phys. Chem. Miner. $28,35-43$.

Kung, J., Weidner, D.J., Li, B., Liebermann, R.C., 2001b. Determination of the elastic properties at high pressure without pressure scale. Eos Trans., AGU 82, F1383.

Li, B., Chen, G., Gwanmesia, G.D., Liebermann, R.C., 1998. Sound velocity measurements at mantle transition zone conditions of pressure and temperature using ultrasonic interferometry in a multianvil apparatus. In: Manghnani, M.H., Yagi, T. (Eds), Properties of Earth and Planetary Materials at High Pressure and Temperature, Geophysical Monograph 101. AGU, Washington, DC, pp. 41-61.

Li, B., Vaughan, M.T., Kung, J., Weidner, D.J., 2001. Direct Length Measurement Using X-Radiography for the Determination of Acoustic Velocities at High Pressure and Temperature. NSLS Activity Report, 2, pp. $103-106$.

Luth, W.L., 1993. Measurement and control of intensive parameters in experiments at high pressure in solid-media apparatus. In: Luth, R.W. (Ed.), Experiments at High Pressure and Applications to the Earth's Mantle, Short Course Handbook 21. Mineral Association Canada, Edmonton, Alta., pp. $15-37$. 
Mao, H.K., Xu, J., Bell, P.M., 1986. Calibration of the ruby pressure gauge to 800 kbar under quasihydrostatic conditions. J. Geophys. Res. 91 (B5), 4673-4676.

McSkimin, H.J., 1950. Ultrasonic measurement techniques applicable to small solid specimens. J. Acoust. Soc. Am. 22, 413-418.

Mueller, H.J., Lauterjung, J., Schilling, F.R., Lathe, C., Nover, G., 2002. Symmetric and asymmetric interferometric method for ultrasonic compressional and shear wave velocity measurements in pistoncylinder and multi-anvil high-pressure apparatus. Eur. J. Mineral. 14, 581-589.

Mueller, H.J., Schilling, F.R., Lauterjung, J., Lathe, C., 2003. A standard free pressure calibration using simultaneous XRD and elastic property measurements in a multi-anvil device. Eur. J. Mineral. 15, $865-873$.

Mueller, H.J., Lathe, C., Wunder, B., 2004. Simultaneous determination of elastic and structural properties under simulated mantle conditions using multi-anvil device MAX80. In: Chen, J., Wang, Y., Duffy, T., Shen, G., Dobrzhinetskaya, L. (Eds), Frontiers in High Pressure Research: Geophysical Applications. Elsevier Science, Amsterdam..

Piermarini, G.J., Block, S., Barnet, J.D., Forman, R.A., 1975. Calibration of the pressure dependence of the $\mathrm{R}_{1}$ ruby fluorescence line to 195 kbar. J. Appl. Phys. 46, 2774-2780.

Ruoff, A.L., Lincoln, R.C., Chen, Y.C., 1973. A new method of absolute high pressure determination. J. Phys. D: Appl. Phys. 6, 1295-1306.

Shen, A.H., Reichmann, H.-J., Chen, G., Angel, R.J., Bassett, W.A., Spetzler, H., 1998. GHz ultrasonic interferometry in a diamond anvil cell: P-wave velocities in periclase to $4.4 \mathrm{GPa}$ and $207^{\circ} \mathrm{C}$. In: Manghnani, M.H., Yagi, T. (Eds), Properties of Earth and Planetary Materials at High Pressure and Temperature, Geophysical Monograph 101. AGU, Washington, DC, pp. 71-77.

Shimomura, O., Yamaoka, S., Yagi, T., Wakutsuki, M., Tsuji, K., Kawamura, H., Hamaya, N., Fukunaga, O., Aoki, K., Akimoto, S., 1985. Multi-anvil type X-ray system for synchrotron radiation. In: Minomura, S. (Ed.), Solid State Physics Under Pressure. Terra Sci. Publ. Co., Tokyo, pp. 351-356.

Vaughan, M.T., 1993. In situ X-ray diffraction using synchrotron radiation at high $P$ and $T$ in a multi-anvil device. In: Luth, R.W. (Ed.), Experiments at High Pressure and Applications to the Earth's Mantle, Short Course Handbook 21. Mineral Association Canada, Edmonton, Alta., pp. 95-130.

Yagi, T., 1988. MAX80: large-volume high-pressure apparatus combined with synchrotron radiation. Eos Trans., AGU 69 12, 18-27.

Yoneda, A., Spetzler, H., Getting, I., 1994. Implication of the complete travel time equation of state for a new pressure scale. In: Schmidt, J.W., Shaner, G.A., Samara, G., Ross, M. (Eds), High-Pressure Science and Technology - 1993. Proceedings of International Association of Research Advancement of High Pressure Science and Technology and the American Physical Society. American Institute of Physics, New York, pp. 1609-1612.

Zha, C.-S., Duffy, T.S., Downs, R.T., Mao, H.K., Hemley, R.J., 1998. Brillouin scattering and X-ray diffraction of San Carlos olivine: direct pressure determination to $32 \mathrm{GPa}$. Earth Planet. Sci. Lett. 159, $25-33$.

Zha, C.-S., Mao, H.K., Hemley, R.J., 2000. Elasiticity of MgO and a primary pressure scale to 55 GPa. Proc. Natl Acad. Sci. 97, 13494-13499.

Zinn, P., Hinze, E., Lauterjung, J., Wirth, R., 1997. Kinetic and microstructural studies of the quartz-coesite phase transition. Phys. Chem. Earth 22, 105-111. 